Trends Cancer. 2015 September 1; 1(1): 53-65. doi:10.1016/j.trecan.2015.07.001.

\title{
Targeting Transcription Factors in Cancer
}

\author{
Anand S. Bhagwat and Christopher R. Vakoc ${ }^{*}$ \\ Cold Spring Harbor Laboratory, 1 Bungtown Road, Cold Spring Harbor, NY 11724, USA
}

\begin{abstract}
Transcription factors (TFs) are commonly deregulated in the pathogenesis of human cancer and are a major class of cancer cell dependencies. Consequently, targeting of TFs can be highly effective in treating particular malignancies, as highlighted by the clinical efficacy of agents that target nuclear hormone receptors. In this review we discuss recent advances in our understanding of TFs as drug targets in oncology, with an emphasis on the emerging chemical approaches to modulate TF function. The remarkable diversity and potency of TFs as drivers of cell transformation justifies a continued pursuit of TFs as therapeutic targets for drug discovery.
\end{abstract}

\section{Introduction}

DNA-binding TFs regulate gene expression by influencing RNA polymerase activity in a gene-specific manner. To carry out this function, TFs minimally employ two distinct interaction surfaces: a sequence-specific DNA-binding domain (e.g., zinc finger, homeodomain, basic helix-loop-helix) and an activation/repression domain that interacts with various cofactors. In eukaryotes, TF cofactors include large, multisubunit protein complexes that either directly activate RNA polymerase II or modify local chromatin structure to allow an increased rate of transcription [1]. TFs regulate many of their target genes by occupying distal enhancer DNA elements, which loop over large genomic distances to regulate target gene promoters [2]. The human genome encodes over 2000 different TFs, many of which are expressed in a cell type-specific manner to coordinate gene expression programs underlying a vast array of cellular processes [2].

Deregulation of TFs is a pervasive theme across many, if not all, forms of human cancer [2]. In tumor cells, genes encoding TFs are often amplified, deleted, rearranged via chromosomal translocation, or subjected to point mutations that result in a gain- or loss-offunction [3]. As prominent examples, TP53 and MYC, which encode the TFs p53 (tumor protein 53) and c-Myc, are among the most commonly altered genes across all cancers [4,5]. Cancer genome studies have also revealed that mutation of TF cofactors (e.g., SWI/SNF, p300/CBP, and Mediator) is also a major mechanism of tumorigenesis [6]. Furthermore, many oncogenic signal transduction cascades alter the function of downstream TFs to

"Correspondence: vakoc@cshl.edu.

Publisher's Disclaimer: This is a PDF file of an unedited manuscript that has been accepted for publication. As a service to our customers we are providing this early version of the manuscript. The manuscript will undergo copyediting, typesetting, and review of the resulting proof before it is published in its final citable form. Please note that during the production process errors may be discovered which could affect the content, and all legal disclaimers that apply to the journal pertain. 
implement gene expression changes that drive cell transformation [3]. The myriad mechanisms of TF deregulation in cancer highlight the centrality of aberrant gene expression in cellular transformation and justify consideration of TFs as therapeutic targets in these diseases.

We discuss below recent insights into the therapeutic strategies to modulate TFs in cancer, and emphasize agents with established clinical efficacy or with promising effects in preclinical models. This review naturally covers the agents that exploit ligand-binding domains (LBDs) of nuclear hormone receptors (NHRs), which are among the most successful targeted therapies in all of oncology. We also highlight some of the emerging chemical strategies to target oncogenic TFs outside the NHR family, which includes methods to stabilize or degrade TFs or interfere with the function of cofactors.

\section{Pharmacological Induction or Restoration of TF Function as a Cancer Therapy}

One established means of undermining the cancer cell state is by pharmacologically elevating the function of specific TFs, in particular those related to tumor-suppressor pathways. In addition, chemical approaches have been devised that restore physiological transcriptional regulation to bypass the aberrant properties of genetically altered TFs or cofactors.

\section{Reversal of PML-RARA-Mediated Repression with all-trans Retinoic Acid (ATRA)}

PML (promyelocytic leukemia)-RARA (retinoic acid receptor $\alpha$ ) is an oncogenic fusion TF produced as a result of the $t(15 ; 17)$ translocation, which occurs in over $98 \%$ of acute promyelocytic leukemias (APLs) [7]. This TF retains an N-terminal zinc-finger region of the PML protein and the DNA-binding domain and LBD of RARA [8]. The fusion protein undergoes aberrant dimerization and assembly with corepressor complexes to deregulate the normal function of RARA, thereby blocking myeloid differentiation (Figure 1) [7]. Empirical evaluation of drug sensitivity led to the discovery that APL cells were highly sensitive to treatment with various retinoid ligands of RARA, including all-trans retinoic acid (ATRA) [9]. This finding was remarkable because the therapeutic activity of ATRA was discovered before the identification of PML-RARA as the causative oncogene in this disease [10,11]. Notably, combination therapy using ATRA and $\mathrm{A}_{2} \mathrm{O}_{3}$ (the latter discussed below) cures more that $90 \%$ of APL patients [12].

Early work demonstrated that the interaction of ATRA with the LBD of PML-RARA leads to a conformational change that disrupted its association with histone deacetylase-containing corepressors in favor of acetyltransferase coactivators [13]. Hence, one outcome of ATRA binding to PML-RARA is its conversion from a repressor to an activator of transcription through the elevation of local histone acetylation at sites occupied by PML-RARA $[7,13,14]$. Because many of the genes repressed by PML-RARA have roles in promoting myeloid differentiation, the net effect of ATRA is the induction of terminal neutrophil differentiation of leukemic blasts (Figure 1) [14]. 


\section{Chemical Restoration of CBF $\beta-R U N X 1$ Complexes in inv(16) Acute Myeloid Leukemia $(\mathrm{AML})$}

Another subtype of acute leukemia is characterized by an inversion on chromosome 16 that fuses core binding factor $\beta(\mathrm{CBF} \beta)$ with the smooth-muscle myosin heavy chain (SMMHC) [15]. CBF $\beta$ is a cofactor for the RUNX (Runt-related) family of TFs, and CBF $\beta-$ RUNX1 heterodimers perform essential roles in normal hematopoiesis [16]. The CBF $\beta-S M M H C$ fusion forms aberrant oligomers that sequester RUNX1 away from the wild-type form of $\mathrm{CBF} \beta$, thereby resulting in leukemogenesis [17]. A recent study employed a high-throughput chemical screen for molecules that disrupt complexes of CBF $\beta-S M M H C$ and RUNX1 using a FRET (fluorescence resonance energy transfer)-based approach [15]. While the lead molecule identified from the screen exhibited moderate potency and a lack of selectivity for the fusion protein, the authors sought to selectively inhibit the oligomeric CBF $\beta-$ SMMHC by generating bivalent derivatives of the compound, named AI-10-49 (Figure 2) [15]. Exposing inv(16) AML cells to AI-10-49 led to the selective disruption of CBF $\beta-S M M H C$ interactions with RUNX1 and restored the formation of wild-type CBF $\beta-R U N X 1$ heterodimers (Figure 2) [15]. Notably, only AML cell lines and patient samples harboring CBF $\beta-$ SMMHC were sensitive to AI-10-49, and this molecule led to impressive survival benefit in a genetically engineered mouse model of this disease [15]. This study highlights how bivalent molecules can be utilized to selectively target an aberrantly oligomerized transcriptional cofactor to restore the formation of physiological TF-cofactor interactions.

\section{Ligand-Induced Activation of the Glucocorticoid Receptor (GR) Leads to Apoptosis in Lymphoid Cancers}

Glucocorticoids are steroid hormones that exert pleiotropic, stress-associated effects on multiple human tissues by activating the function of GR [18]. One of the effects of GR activation with synthetic agonists (e.g., dexamethasone and prednisolone) is a severe defect in the proliferation and survival of lymphocytes, which has led to a widespread use of glucocorticoids as immunosuppressants and as a therapy for acute lymphoblastic leukemia (ALL) and other lymphoid neoplasms (Figure 3) [19,20]. Part of the mechanism underlying the induction of lymphocyte apoptosis by glucocorticoids is the role of GR in regulating the expression of genes encoding the BCL2 (B cell lymphoma 2) family of apoptosis regulators $[21,22]$. Indeed, GR activates expression of the proapoptotic BIM (BCL2 interacting mediator of cell death) gene, which is required for glucocorticoid-induced apoptosis of transformed lymphoid cells [21,22]. The clinical use of glucocorticoids in lymphoid cancers illustrates how chemical augmentation of TF function can cause lethality to cancer cells in a manner specific to the cell lineage from which the tumor is derived.

Despite the well-established efficacy of GR modulation in lymphoid cancers, the clinical response to glucocorticoids is heterogeneous and limited by the emergence of resistance. It has been found that GR occupancy at an intronic BIM enhancer occurs in dexamethasonesensitive ALLs, but not in dexamethasone-resistant ALL, highlighting how TF genomic occupancy can be utilized as a therapeutic response biomarker [23]. In a separate study it was found that increased expression of caspase 1 confers resistance to glucocorticoids via caspase 1-mediated cleavage of GR to antagonize its transcriptional output [24]. Resistance to glucocorticoid therapy can also be conferred via the genetic loss of PTEN (phosphatase 
and tensin homology), which leads to AKT (protein kinase B)-mediated

hyperphosphorylation of GR to block its nuclear entry [25]. Loss-of-function mutations or transcriptional silencing of GR coactivators (e.g., CBP and SWI/SNF) has also been associated with dexamethasone resistance in ALL [26,27]. Collectively, these studies raise the possibility that targeting of caspase 1 , AKT, or GR cofactors could improve the efficacy of glucocorticoid therapy in ALL.

\section{Stabilization of p53 by Antagonizing MDM2}

Mutations that inactivate TP53 are among the most frequent genetic alterations in human cancer. However, many cancers retain wild-type TP53, but depend on E3 ligase protein MDM2 (double minute 2) for ubiquitin-mediated destruction of p53 via the proteasome, or on MDMX/MDM4, which directly binds to and inhibits the function of p53 [28]. Because genetic restoration of p53 function can lead to profound tumor regressions in animal models, several groups have developed MDM2 inhibitors as targeted therapy for TP53 wild-type cancers [29-31]. The first of such inhibitors, nutlins, were identified via a high-throughput chemical screen for molecules that disrupted the binding of MDM2 and p53 [32]. Preclinical studies with nutlins have demonstrated strong effects in suppressing tumor growth in mouse xenografts via induction of p53-mediated apoptosis and several molecules based on the nutlin chemical structure are currently in clinical development [28,31].

\section{Targeting of Lineage-Specific Non-Oncogene TF Dependencies}

While genetically altered TFs, such c-Myc and PML-RARA, represent compelling targets, non-oncogene TFs that support growth of discrete tissues can also be suitable for therapeutic intervention in cancer. The unique requirement for the estrogen receptor a (ERa) and the androgen receptor (AR) for the growth of reproductive tissues has afforded the development of highly-effective and well-tolerated cancer therapeutics in breast and prostate cancer, respectively.

\section{ER in Breast Cancer}

Approximately $70 \%$ of breast cancers rely on estrogen for sustained cell growth, a feature that cancer cells share with the ductal epithelium cell-of-origin [33,34]. These effects are largely mediated through the interaction of estrogen with the LBD of a single NHR, ERa $[33,35]$. While not an oncogene per se, a subset of breast cancers utilize ER to sustain their aberrant capabilities. Notably, genetic knockout of ER in mice is compatible with viability, but animals display abnormalities in the development of female reproductive organs (uterus, ovaries, and mammary glands) and other organ systems (e.g., diminished bone mineralization) [36]. Nonetheless, the tissue context-specific ER requirement for cell proliferation across adult tissues has provided a wide therapeutic window for ER modulation as a therapeutic approach in breast cancer.

Several pharmacological strategies are available to target the ER LBD to suppress its function [37]. Selective estrogen receptor modulators (SERMs), such as tamoxifen and raloxifene, directly compete with estrogen for binding to the ER LBD [38]. Other ER antagonists such as fulvestrant bind to the ER LBD at much higher affinity than SERMs and 
cause ER degradation [39,40]. Another route to ER inhibition is aimed at reducing the availability of estrogen by inhibiting aromatase, an enzyme that converts circulating androgens into estrogen [41]. The net effect of each of these therapies is a selective block in cell cycle progression and the induction of programmed cell death, which can lead to tumor regression in vivo while having an acceptable side-effect profile in other tissues (Figure 4A) $[37,42]$.

One remarkable feature of ER revealed through epigenomic approaches is its capacity to rearrange its global genomic occupancy, or cistrome, in response to various stimuli $[35,43]$. Chromatin immunoprecipitation combined with high-throughput sequencing (ChIP-seq) analysis in primary human breast tumors has revealed a unique ER cistrome in poor-versus favorable-prognosis tumors. These ER cistromes are specified in part through the actions of the pioneer TF FOXA1 (forkhead box A1), which can render enhancers competent for ER occupancy $[44,45]$. Signaling cascades downstream of HER2 (epidermal growth factor receptor 2/ERBB2), an oncogene amplified in $\sim 20 \%$ of breast cancers, can also reprogram ER cistromes and attenuate the sensitivity of breast cancer cells to tamoxifen [46,47]. Similarly, inflammation within the tumor microenvironment is associated with resistance to tamoxifen therapy and remodeling of ER occupancy at enhancers, which can be mediated by tumor necrosis factor $a(\mathrm{TNFa})$-dependent activation of nuclear factor $\kappa b(\mathrm{NF}-\kappa \mathrm{B})[48,49]$. Copy-number amplifications of ER-bound enhancers at 17q23 and 20q13 have also been associated with tamoxifen resistance, which may provide a genetic means of reprogramming ER genomic occupancy [50]. Collectively, these studies highlight how TF cistromes can be dynamically altered to dictate the transcriptional output and overall efficacy of TFmodulating therapeutics.

\section{AR in Prostate Cancer}

Androgens, such as testosterone and dihydrotestosterone, are steroid hormones that perform essential roles in the development of the male reproductive system, including a role in promoting growth of normal prostate epithelium [51]. Similar to the estrogen dependence of breast cancers, tumors derived from prostate epithelium rely on endogenous androgens for disease maintenance and progression [52]. This finding has motivated numerous strategies to deplete or block the effect of androgens as a therapeutic approach for men with advanced prostate cancer. The majority of the effects of androgens on human biology is mediated by AR. In prostate cancer cells, the interaction of androgens with the LBD of AR leads to transcriptional changes at an array of downstream target genes involved in the control of cell proliferation and survival (Figure 4B) [53,54].

Androgen production can be suppressed surgically by orchiectomy or pharmacologically by targeting the hypothalamic-pituitary axis with luteinizing hormone releasing hormone (LHRH) analogs (e.g., leuprolide) or by blocking the enzymatic activity of cytochrome P450 CYPC17 (e.g., with abiraterone) [55-57]. Another class of drugs block the effects of androgens through direct antagonism of AR function. First generation anti-androgens (e.g., flutamide and bicalutamide) and second-generation anti-androgens (enzalutamide and ARN-509) compete with endogenous androgens for binding to the AR LBD [53,58,59]. Second generation anti-androgens exhibit enhanced affinity for AR and have overcome 
several of the common resistance mechanisms associated with first-generation agents $[53,60]$. However, AR point mutations and alterations of AR splice variants have emerged as resistance mechanisms that also limit the efficacy of second-generation anti-androgens $[58,61-64]$.

Analysis of AR genomic occupancy has yielded significant insights into mechanisms of prostate cancer progression and resistance to anti-androgen therapy [54]. One of the major determinants of AR cistromes in prostate cancer is the ETS (avian erythroblastosis virus E26 oncogene homolog) family of TFs, which are genetically altered and overexpressed in this disease $[65,66]$. Depending on the cellular context, ETS factors can either repress or support the output of AR signaling [65,66]. Recent evidence also suggests that the essential function of AR in advanced prostate cancer can be replaced by GR, thereby allowing a mechanism of glucocorticoid-dependent resistance to anti-androgen therapy [67]. In such tumors, it was shown that GR invades the AR cistrome to maintain a parallel transcriptional pathway that supports disease progression [67].

\section{Targeting of TF Cofactors}

The regulatory function of TFs is reliant on direct interactions with a vast assortment of transcriptional cofactors as a means of influencing RNA polymerase II activity. Hence, many TF-cofactor interaction surfaces represent compelling therapeutic targets. While historically such chemical approaches have been limited by a lack of potency, a few smallmolecule or short peptide-based approaches that target cofactor interactions have been shown to suppress oncogenic TF function and exhibit promising therapeutic effects in preclinical cancer models.

\section{NOTCH1-MAML in T cell leukemia (T-ALL)}

$\mathrm{NOTCH} \mathrm{l}$ encodes a transmembrane protein that is cleaved upon binding to extracellular ligands to allow translocation of its intracellular domain (ICN1) to the nucleus, where it serves as cofactor for the TF CSL ('CBF1, suppressor of hairless, Lag-1') [68]. The ICN1CSL interaction creates a docking site for the mastermind-like (MAML) family of coactivators, which together promote the transcriptional output to NOTCH1 signaling [68]. Notably, NOTCH1 mutations are found in T-ALL that increase the nuclear localization of ICN1 to promote leukemia initiation [68]. As a potential treatment strategy for NOTCH1driven leukemia, Moellering et al. developed a cell-permeable stabilized a-helical peptide derived from MAML1 (SAHM1), which binds to ICN1-CSL with a Kd of $120 \mathrm{nM}$ to competitively block the association of endogenous MAML1 with ICN1 [69]. Treatment of NOTCH1-mutant T-ALL (T-acute lymphoblastic leukemia) cells with SAHM1 attenuated NOTCH1-dependent transcriptional signatures and inhibited T-ALL progression in xenograft models [69].

\section{BCL6-SMRT in B Cell Lymphoma}

BCL6 is an oncogenic zinc-finger TF that is often overexpressed in B cell lymphomas as a consequence of BCL6 chromosomal translocations or promoter mutations [70]. BCL6 represses its downstream target genes via a BTB domain which interacts directly with a 
variety of corepressors, including SMRT (silencing mediator of retinoic acid and thyroid hormone receptor) [70]. To devise a means of disrupting BCL6-mediated repression, a structure-based in silico drug design effort led to the identification of a small molecule, 79-6, that binds to the SMRT interaction site of the BCL6 BTB domain with a Kd of 125 $\mu \mathrm{M}$ [71]. 79-6 can disrupt SMRT-BCL6 interactions in lymphoma cells and leads to upregulation of BCL6 target genes [71]. Despite the moderate affinity for BCL6, treatment of lymphoma-bearing mice with 79-6 led to diminished tumor growth in vivo [71].

\section{Blockade of TF Output by Chemical Inhibition of BRD4}

An example of a general TF cofactor is the bromodomain and extraterminal (BET) protein BRD4, which can interact directly with a vast array of TFs in a bromodomain-dependent or independent manner [72]. Moreover, BRD4 bromodomains can also bind to acetylated nucleosomes that lie adjacent to TF-occupied enhancers and promoter, thus allowing BRD4 to promote TF-mediated activation even in the absence of direct TF interactions [72]. Inhibition of BET bromodomains with potent small-molecule inhibitors (e.g., JQ1 or IBET) has been shown to suppress the functional output of a vast array of oncogenic TFs, including ERG (ETS-related gene), c-Myb, c-Myc, E2F1 (E2F transcription factor 1), and NF- $\kappa B$ [7377]. Moreover, several oncogenic loci exhibit massive enrichment of BRD4 at distal enhancer elements, termed super-enhancers, which may also confer cancer cell hypersensitivity to BRD4 inhibition [78]. Chemical inhibition of BET bromodomains has been shown to suppress tumor progression in a wide range of animal models while, remarkably, exhibiting minimal toxicity to normal tissues [72,76]. BET inhibitors are currently under investigation as cancer therapeutics in several early-stage clinical trials.

One of the impressive effects of BRD4 inhibition is its capacity to suppress the expression of genes encoding oncogenic TFs, including MYC [79,80]. If such effects can occur with a degree of specificity, such an approach provides an attractive indirect route for TF modulation. While not a TF cofactor per se, the histone H3 lysine 79 (H3K79) methyltransferase DOT1L (DOT1-like) is essential to maintain expression of essential homeodomain-containing TFs (e.g., HOXA9) in AML [81]. Furthermore, small-molecule inhibitors of DOT1L suppress HOXA9 expression and leukemia progression in animal models, which has led clinical trials evaluating these agents in leukemia patients [81]. Chemical inhibition of the CDK7 (cyclin-dependent kinase 7) subunit of TFIIH (transcription factor II human) has also been shown to disproportionately suppress the expression of lineage-specific TFs in various cancer models in association with therapeutic benefit [82].

\section{Targeting TFs for Proteasome-Mediated Destruction}

An alternative approach to disrupting TF-DNA and TF-cofactor interactions with smallmolecules is to target TFs for proteolysis, an approach that has been associated with remarkable clinical efficacy in the examples highlighted below. 


\section{Targeting PML-RARA for Destruction with $\mathrm{As}_{2} \mathrm{O}_{3}$ and ATRA}

The mechanism underlying the curative effects of $\mathrm{As}_{2} \mathrm{O}_{3}$ and ATRA in APL has been the subject of intense investigation in recent years. Despite its identification as therapeutic via an empirical evaluation, $\mathrm{As}_{2} \mathrm{O}_{3}$ has been shown to induce PML-RARA degradation by directly interacting with the PML moiety of the fusion TF [83,84]. In this context, arsenic can replace the zinc ion that normally interacts with PML [84]. This leads to oxidation of vicinal cysteine residues and enhances the oligomerization of PML-RAR, which in turn increases its interaction with the SUMO (small ubiquitin-like modifier)-conjugating enzyme UBC9 ('ubiquitin-conjugating enzyme 9') [83,84]. This results in PML-RARA sumoylation and subsequent RNF4 (ring finger protein 4)-mediated polyubiquitination, ultimately leading to destruction of the oncoprotein [83-86]. This example highlights how pharmacological TF degradation can be a highly-selective and effective therapeutic approach in treating lethal malignancies.

ATRA reverses the transcriptional repression mechanism of PML-RARA to cause APL differentiation; however, earlier work had shown that ATRA also triggers the degradation of PML-RARA [87]. The latter effect has been suggested to occur as an indirect consequence of ATRA interacting with the prolyl isomerase PIN1 (peptidylprolyl cis/trans isomerase 1), which in turn regulates PML-RARA stability [88]. Synthetic retinoid molecules have recently been identified that potently antagonize PML-RARA-mediated repression, but without altering PML-RARA levels [89]. Although these retinoids induce differentiation of APL cells to a comparable extent to ATRA, these compounds are much less active in eradicating leukemia in mice [89]. This suggests that differentiation is not the underlying mechanism relevant to the curative effects of ATRA in APL [89]. It now appears that PMLRARA destruction via ATRA or $\mathrm{As}_{2} \mathrm{O}_{3}$ leads to eradication of leukemia stem cells via induction of p53-mediated senescence, which leads to curative effects in this disease [90].

\section{Targeting TFs or Cofactors for Destruction using Phthalimide-Based Drugs}

Phthalimide-based drugs (thalidomide, lenalidomide, and pomalidomide) were originally withdrawn from clinical use because of their teratogenicity, but were later realized to exhibit potent therapeutic effects in multiple myeloma and myelodysplasia. The molecular target of phthalimides was recently discovered to be the cullin-RING ubiquitin ligase protein cereblon [91]. To reveal the relevant cereblon substrates whose levels are modulated by phthalimides, two independent groups carried out proteomic screens that led to the identification of Ikaros-family TFs, IKZF1 and IKZF3, as the cereblon substrates modulated by phthalimide treatment of multiple myeloma cells $[92,93]$. Binding of phthalimides to cereblon triggered polyubiquitylation of IKZF1/3 and subsequent proteasome-mediated degradation (Figure 5) [92,93]. Structural studies suggest that the interaction of thalidomide with cereblon may create a neomorphic interface for IKZF1/3 that enhances substrate recognition, potentially at the expense of natural cereblon substrates [94,95]. IKZF1/3 are essential TFs in the B cell lineage, and mutation of the cereblon degron motifs found on these TFs was sufficient to render myeloma cells resistant to phthalimides [92,93]. In analogy to $\mathrm{As}_{2} \mathrm{O}_{3}$ and ATRA in APL, these studies demonstrate how an empirically identified cancer therapy was subsequently realized to induce TF destruction as part of its mechanism of action. 
The aforementioned successes of small-molecule-based targeting of TFs for proteolysis raises the question as to the whether such an approach can be generalized to target other intractable proteins. A recent study has now shown that various small molecules can be conjugated to the aryl ring of thalidomide to cause cereblon-mediated degradation of their interacting proteins [96]. This was shown using the BET bromodomain inhibitor JQ1, which when linked to thalidomide led to potent suppression of BET protein levels and enhanced anti-leukemia effects [96]. An independent series of thalidomide conjugates were also able to cause degradation of FKBP12 (FK506 binding protein 12) [96]. This study reveals a powerful chemical tool that could be employed for targeting oncogenic TFs or their cofactors. Traditional chemical approaches require high-affinity and high-specificity interactions with functionally-important surfaces to interfere with their targets. Phthalamide conjugation would presumably allow small molecules to suppress their protein targets without necessarily binding to functionally important surfaces, which has potential for expanding the number of targetable proteins.

\section{Concluding Remarks}

Our understanding of the clinical efficacy and current limitations of NHR modulation in cancer can greatly aid our assessment of other TF classes as candidates for drug development (Outstanding Questions). One important lesson learned from the agents that target AR and ER is that a therapeutic window need not rely on the targeting of a genetically altered TF functionality. Targeting of ER and AR instead exploits a lineage-specific, nononcogene dependency on these regulators that is inherited from the cell of origin. A large number of TFs are also required in discrete cell types, and hence targeting of such regulators could have an acceptable risk-to-benefit ratio, depending on the essentiality of the tissue of origin. NHR modulation has also provided fundamental insights into the modes of resistance that can emerge upon suppressing a single TF dependency, such as mutations of the TFs themselves, mutations of cofactors, and remodeling of TF cistromes.

One theme that emerges from this review is that chemical modulation of TFs can be achieved through remarkably diverse, and often empirical, strategies. The clinical success of agents such as $\mathrm{As}_{2} \mathrm{O}_{3}$ and thalidomide underscores how small molecules can target TFs for proteasome-mediated destruction in a highly-selective manner. This represents an alternative to the chemical disruption of TF-cofactor or TF-DNA interactions, which can be limited by a lack of potency [97]. High-throughput cell-based chemical screens that evaluate for TF degradation may provide a means to develop agents that suppress other elusive TF dependencies in oncology, such as c-Myc. Conversely, TFs can also be stabilized pharmacologically by blocking their destruction, as in the case of drugs that block MDM2/ MDMX-mediated targeting of p53 [32]. Finally, TFs can also be suppressed at the transcriptional level by targeting of general factors such as BRD4, DOT1L, and CDK7 $[72,82]$.

Recent advances in functional genomics, such as the implementation of CRISPR (clustered regularly interspaced short palindromic repeats)-based genetic screens, will soon provide a comprehensive picture of TF dependencies across diverse forms of cancer [98-100]. The context-specific nature of many TF dependencies is likely to lead to the identification of a 
large number of therapeutic opportunities. However, to exploit TFs as drug targets demands a deep biochemical understanding of TF-cofactor interactions and the mechanisms that control TF protein stability. Basic research that investigates mechanisms of TF function in normal and cancer contexts will be crucial to advance this important area of translational cancer research.

\section{Acknowledgments}

A.S.B. is supported by the National Cancer Institute (grant F30 CA186632). C.R.V. is supported by the National Institutes of Health (grant CA174793) and a Burroughs-Wellcome Fund Career Award for Medical Scientists.

\section{References}

1. Yan C, Higgins PJ. Drugging the undruggable: transcription therapy for cancer. Biochim Biophys Acta. 2013; 1835:76-85. [PubMed: 23147197]

2. Lee TI, Young RA. Transcriptional regulation and its misregulation in disease. Cell. 2013; 152:1237-1251. [PubMed: 23498934]

3. Darnell JE Jr. Transcription factors as targets for cancer therapy. Nat Rev Cancer. 2002; 2:740-749. [PubMed: 12360277]

4. Lee EY, Muller WJ. Oncogenes and tumor suppressor genes. Cold Spring Harb Perspect Biol. 2010; 2:a003236. [PubMed: 20719876]

5. Bretones G, et al. Myc and cell cycle control. Biochim Biophys Acta. 2015; 1849:506-516. [PubMed: 24704206]

6. Garraway LA, Lander ES. Lessons from the cancer genome. Cell. 2013; 153:17-37. [PubMed: 23540688]

7. Rice KL, de The H. The acute promyelocytic leukaemia success story: curing leukaemia through targeted therapies. J Intern Med. 2014; 276:61-70. [PubMed: 24635409]

8. de The $\mathrm{H}$, et al. The $\mathrm{t}(15 ; 17)$ translocation of acute promyelocytic leukaemia fuses the retinoic acid receptor alpha gene to a novel transcribed locus. Nature. 1990; 347:558-561. [PubMed: 2170850]

9. Wang ZY, Chen Z. Acute promyelocytic leukemia: from highly fatal to highly curable. Blood. 2008; 111:2505-2515. [PubMed: 18299451]

10. Huang ME, et al. Use of all-trans retinoic acid in the treatment of acute promyelocytic leukemia. Blood. 1988; 72:567-572. [PubMed: 3165295]

11. Huang ME, et al. All-trans retinoic acid with or without low dose cytosine arabinoside in acute promyelocytic leukemia. Report of 6 cases. Chin Med J (Engl). 1987; 100:949-953. [PubMed: 3133168]

12. Lo-Coco F, et al. Retinoic acid and arsenic trioxide for acute promyelocytic leukemia. N Engl J Med. 2013; 369:111-121. [PubMed: 23841729]

13. Lin RJ, et al. Role of the histone deacetylase complex in acute promyelocytic leukaemia. Nature. 1998; 391:811-814. [PubMed: 9486654]

14. Martens JH, et al. PML-RARalpha/RXR alters the epigenetic landscape in acute promyelocytic leukemia. Cancer cell. 2010; 17:173-185. [PubMed: 20159609]

15. Illendula A, et al. A small-molecule inhibitor of the aberrant transcription factor CBFbetaSMMHC delays leukemia in mice. Science. 2015; 347:779-784. [PubMed: 25678665]

16. de Bruijn MF, Speck NA. Core-binding factors in hematopoiesis and immune function. Oncogene. 2004; 23:4238-4248. [PubMed: 15156179]

17. Lukasik SM, et al. Altered affinity of CBF beta-SMMHC for Runx1 explains its role in leukemogenesis. Nature structural biology. 2002; 9:674-679. [PubMed: 12172539]

18. Nicolaides NC, et al. The human glucocorticoid receptor: molecular basis of biologic function. Steroids. 2010; 75:1-12. [PubMed: 19818358]

19. Inaba H, Pui CH. Glucocorticoid use in acute lymphoblastic leukaemia. Lancet Oncol. 2010; 11:1096-1106. [PubMed: 20947430] 
20. Pui CH, Evans WE. Treatment of acute lymphoblastic leukemia. N Engl J Med. 2006; 354:166178. [PubMed: 16407512]

21. Wang Z, et al. Microarray analysis uncovers the induction of the proapoptotic BH3-only protein Bim in multiple models of glucocorticoid-induced apoptosis. J Biol Chem. 2003; 278:2386123867. [PubMed: 12676946]

22. Abrams MT, et al. Inhibition of glucocorticoid-induced apoptosis by targeting the major splice variants of BIM mRNA with small interfering RNA and short hairpin RNA. J Biol Chem. 2004; 279:55809-55817. [PubMed: 15509554]

23. Jing D, et al. Opposing regulation of BIM and BCL2 controls glucocorticoid-induced apoptosis of pediatric acute lymphoblastic leukemia cells. Blood. 2015; 125:273-283. [PubMed: 25336632]

24. Paugh SW, et al. NALP3 inflammasome upregulation and CASP1 cleavage of the glucocorticoid receptor cause glucocorticoid resistance in leukemia cells. Nat Genet. 2015; 47:607-614. [PubMed: 25938942]

25. Piovan E, et al. Direct reversal of glucocorticoid resistance by AKT inhibition in acute lymphoblastic leukemia. Cancer cell. 2013; 24:766-776. [PubMed: 24291004]

26. Mullighan CG, et al. CREBBP mutations in relapsed acute lymphoblastic leukaemia. Nature. 2011; 471:235-239. [PubMed: 21390130]

27. Pottier N, et al. The SWI/SNF chromatin-remodeling complex and glucocorticoid resistance in acute lymphoblastic leukemia. J Natl Cancer Inst. 2008; 100:1792-1803. [PubMed: 19066270]

28. Duffy MJ, et al. p53 as a target for the treatment of cancer. Cancer treatment reviews. 2014; 40:1153-1160. [PubMed: 25455730]

29. Xue W, et al. Senescence and tumour clearance is triggered by 553 restoration in murine liver carcinomas. Nature. 2007; 445:656-660. [PubMed: 17251933]

30. Ventura A, et al. Restoration of p53 function leads to tumour regression in vivo. Nature. 2007; 445:661-665. [PubMed: 17251932]

31. Zhang Q, et al. Targeting p53-MDM2-MDMX loop for cancer therapy. Sub-cellular biochemistry. 2014; 85:281-319. [PubMed: 25201201]

32. Vassilev LT, et al. In vivo activation of the p53 pathway by small-molecule antagonists of MDM2. Science. 2004; 303:844-848. [PubMed: 14704432]

33. Huang, B., et al. Estrogen receptors in breast carcinogenesis and endocrine therapy. Mol Cell Endocrinol. 2014. Published online November 26, 2014. http://dx.doi.org/10.1016/j.mce. 2014.11.015

34. van Kruchten M, et al. PET imaging of oestrogen receptors in patients with breast cancer. Lancet Oncol. 2013; 14:e465-475. [PubMed: 24079874]

35. Manavathi B, et al. Estrogen receptor coregulators and pioneer factors: the orchestrators of mammary gland cell fate and development. Front Cell Dev Biol. 2014; 2:34. [PubMed: 25364741]

36. Lee HR, et al. Functions and physiological roles of two types of estrogen receptors, ERalpha and ERbeta, identified by estrogen receptor knockout mouse. Laboratory animal research. 2012; 28:71-76. [PubMed: 22787479]

37. Mehta RS, et al. Combination anastrozole and fulvestrant in metastatic breast cancer. $\mathrm{N}$ Engl $\mathbf{J}$ Med. 2012; 367:435-444. [PubMed: 22853014]

38. Komm BS, Mirkin S. An overview of current and emerging SERMs. J Steroid Biochem Mol Biol. 2014; 143:207-222. [PubMed: 24667357]

39. Howell A. Pure oestrogen antagonists for the treatment of advanced breast cancer. Endocr Relat Cancer. 2006; 13:689-706. [PubMed: 16954425]

40. Wijayaratne AL, McDonnell DP. The human estrogen receptor-alpha is a ubiquitinated protein whose stability is affected differentially by agonists, antagonists, and selective estrogen receptor modulators. J Biol Chem. 2001; 276:35684-35692. [PubMed: 11473106]

41. Goss PE, et al. Exemestane for breast-cancer prevention in postmenopausal women. N Engl J Med. 2011; 364:2381-2391. [PubMed: 21639806]

42. Scherbakov AM, et al. Molecular mechanisms of hormone resistance of breast cancer. Bull Exp Biol Med. 2013; 155:384-395. [PubMed: 24137610] 
43. Tang Q, et al. A comprehensive view of nuclear receptor cancer cistromes. Cancer research. 2011; 71:6940-6947. [PubMed: 21940749]

44. Ross-Innes CS, et al. Differential oestrogen receptor binding is associated with clinical outcome in breast cancer. Nature. 2012; 481:389-393. [PubMed: 22217937]

45. Carroll JS, et al. Chromosome-wide mapping of estrogen receptor binding reveals long-range regulation requiring the forkhead protein FoxA1. Cell. 2005; 122:33-43. [PubMed: 16009131]

46. Lupien M, et al. Growth factor stimulation induces a distinct ER(alpha) cistrome underlying breast cancer endocrine resistance. Genes \& development. 2010; 24:2219-2227. [PubMed: 20889718]

47. Ferreira AR, et al. Treatment of early-stage HER $2^{+}$breast cancer-an evolving field. Ecancermedicalscience. 2015; 9:523. [PubMed: 25932047]

48. Osborne CK, Schiff R. Mechanisms of endocrine resistance in breast cancer. Annu Rev Med. 2011; 62:233-247. [PubMed: 20887199]

49. Franco HL, et al. TNFalpha signaling exposes latent estrogen receptor binding sites to alter the breast cancer cell transcriptome. Molecular cell. 2015; 58:21-34. [PubMed: 25752574]

50. Hsu PY, et al. Amplification of distant estrogen response elements deregulates target genes associated with tamoxifen resistance in breast cancer. Cancer cell. 2013; 24:197-212. [PubMed: 23948299]

51. Shen MM, Abate-Shen C. Molecular genetics of prostate cancer: new prospects for old challenges. Genes \& development. 2010; 24:1967-2000. [PubMed: 20844012]

52. Huggins C, et al. Studies on prostatic cancer. II. The effects of castration on advanced carcinoma of the prostate gland. Archives of Surgery. 1941; 43:209-223.

53. Carver BS. Strategies for targeting the androgen receptor axis in prostate cancer. Drug Discov Today. 2014; 19:1493-1497. [PubMed: 25107669]

54. Sharma NL, et al. The androgen receptor induces a distinct transcriptional program in castrationresistant prostate cancer in man. Cancer cell. 2013; 23:35-47. [PubMed: 23260764]

55. Moul JW. Utility of LHRH antagonists for advanced prostate cancer. Can J Urol. 2014; 21:22-27. [PubMed: 24775720]

56. de Bono JS, et al. Abiraterone and increased survival in metastatic prostate cancer. N Engl J Med. 2011; 364:1995-2005. [PubMed: 21612468]

57. Ryan CJ, et al. Abiraterone in metastatic prostate cancer without previous chemotherapy. N Engl J Med. 2013; 368:138-148. [PubMed: 23228172]

58. Wyatt AW, Gleave ME. Targeting the adaptive molecular landscape of castration-resistant prostate cancer. EMBO Mol Med. 2015; 20:878-894. [PubMed: 25896606]

59. Aragon-Ching JB. The evolution of prostate cancer therapy: targeting the androgen receptor. Front Oncol. 2014; 4:295. [PubMed: 25386409]

60. Tran C, et al. Development of a second-generation antiandrogen for treatment of advanced prostate cancer. Science. 2009; 324:787-790. [PubMed: 19359544]

61. Joseph JD, et al. A clinically relevant androgen receptor mutation confers resistance to secondgeneration antiandrogens enzalutamide and ARN-509. Cancer Discov. 2013; 3:1020-1029. [PubMed: 23779130]

62. Korpal M, et al. An F876L mutation in androgen receptor confers genetic and phenotypic resistance to MDV3100 (enzalutamide). Cancer Discov. 2013; 3:1030-1043. [PubMed: 23842682]

63. Balbas MD, et al. Overcoming mutation-based resistance to antiandrogens with rational drug design. Elife. 2013; 2:e00499. [PubMed: 23580326]

64. Antonarakis ES, et al. AR-V7 and resistance to enzalutamide and abiraterone in prostate cancer. N Engl J Med. 2014; 371:1028-1038. [PubMed: 25184630]

65. Chen Y, et al. ETS factors reprogram the androgen receptor cistrome and prime prostate tumorigenesis in response to PTEN loss. Nature medicine. 2013; 19:1023-1029.

66. Yu J, et al. An integrated network of androgen receptor, polycomb, and TMPRSS2-ERG gene fusions in prostate cancer progression. Cancer cell. 2010; 17:443-454. [PubMed: 20478527]

67. Arora VK, et al. Glucocorticoid receptor confers resistance to antiandrogens by bypassing androgen receptor blockade. Cell. 2013; 155:1309-1322. [PubMed: 24315100] 
68. Aster JC, et al. Notch signalling in T-cell lymphoblastic leukaemia/lymphoma and other haematological malignancies. The Journal of pathology. 2011; 223:262-273. [PubMed: 20967796]

69. Moellering RE, et al. Direct inhibition of the NOTCH transcription factor complex. Nature. 2009; 462:182-188. [PubMed: 19907488]

70. Hatzi K, Melnick A. Breaking bad in the germinal center: how deregulation of BCL6 contributes to lymphomagenesis. Trends in molecular medicine. 2014; 20:343-352. [PubMed: 24698494]

71. Cerchietti LC, et al. A small-molecule inhibitor of BCL6 kills DLBCL cells in vitro and in vivo. Cancer cell. 2010; 17:400-411. [PubMed: 20385364]

72. Shi J, Vakoc CR. The mechanisms behind the therapeutic activity of BET bromodomain inhibition. Molecular cell. 2014; 54:728-736. [PubMed: 24905006]

73. Roe JS, et al. BET bromodomain inhibition suppresses the function of hematopoietic transcription factors in acute myeloid leukemia. Molecular cell. 2015; 58:1028-1039. [PubMed: 25982114]

74. Chapuy B, et al. Discovery and characterization of super-enhancer-associated dependencies in diffuse large B cell lymphoma. Cancer cell. 2013; 24:777-790. [PubMed: 24332044]

75. Asangani IA, et al. Therapeutic targeting of BET bromodomain proteins in castration-resistant prostate cancer. Nature. 2014; 510:278-282. [PubMed: 24759320]

76. Filippakopoulos P, et al. Selective inhibition of BET bromodomains. Nature. 2010; 468:10671073. [PubMed: 20871596]

77. Nicodeme E, et al. Suppression of inflammation by a synthetic histone mimic. Nature. 2010; 468:1119-1123. [PubMed: 21068722]

78. Loven J, et al. Selective inhibition of tumor oncogenes by disruption of super-enhancers. Cell. 2013; 153:320-334. [PubMed: 23582323]

79. Delmore JE, et al. BET bromodomain inhibition as a therapeutic strategy to target c-Myc. Cell. 2011; 146:904-917. [PubMed: 21889194]

80. Zuber J, et al. RNAi screen identifies Brd4 as a therapeutic target in acute myeloid leukaemia. Nature. 2011; 478:524-528. [PubMed: 21814200]

81. Bernt KM, Armstrong SA. A role for DOT1L in MLL-rearranged leukemias. Epigenomics. 2011; 3:667-670. [PubMed: 22126283]

82. Kwiatkowski N, et al. Targeting transcription regulation in cancer with a covalent CDK7 inhibitor. Nature. 2014; 511:616-620. [PubMed: 25043025]

83. Zhang XW, et al. Arsenic trioxide controls the fate of the PML-RARalpha oncoprotein by directly binding PML. Science. 2010; 328:240-243. [PubMed: 20378816]

84. Jeanne M, et al. PML/RARA oxidation and arsenic binding initiate the antileukemia response of As2O3. Cancer cell. 2010; 18:88-98. [PubMed: 20609355]

85. Lallemand-Breitenbach V, et al. Arsenic degrades PML or PML-RARalpha through a SUMOtriggered RNF4/ubiquitin-mediated pathway. Nat Cell Biol. 2008; 10:547-555. [PubMed: 18408733]

86. Tatham MH, et al. RNF4 is a poly-SUMO-specific E3 ubiquitin ligase required for arsenic-induced PML degradation. Nat Cell Biol. 2008; 10:538-546. [PubMed: 18408734]

87. Zhu J, et al. Retinoic acid induces proteasome-dependent degradation of retinoic acid receptor alpha (RARalpha) and oncogenic RARalpha fusion proteins. Proceedings of the National Academy of Sciences of the United States of America. 1999; 96:14807-14812. [PubMed: 10611294]

88. Wei S, et al. Active Pin1 is a key target of all-trans retinoic acid in acute promyelocytic leukemia and breast cancer. Nature medicine. 2015; 21:457-466.

89. Ablain J, et al. Uncoupling RARA transcriptional activation and degradation clarifies the bases for APL response to therapies. J Exp Med. 2013; 210:647-653. [PubMed: 23509325]

90. Ablain J, et al. Activation of a promyelocytic leukemia-tumor protein 53 axis underlies acute promyelocytic leukemia cure. Nature medicine. 2014; 20:167-174.

91. Ito T, et al. Identification of a primary target of thalidomide teratogenicity. Science. 2010; 327:1345-1350. [PubMed: 20223979]

92. Kronke J, et al. Lenalidomide causes selective degradation of IKZF1 and IKZF3 in multiple myeloma cells. Science. 2014; 343:301-305. [PubMed: 24292625] 
93. Lu G, et al. The myeloma drug lenalidomide promotes the cereblon-dependent destruction of Ikaros proteins. Science. 2014; 343:305-309. [PubMed: 24292623]

94. Fischer ES, et al. Structure of the DDB1-CRBN E3 ubiquitin ligase in complex with thalidomide. Nature. 2014; 512:49-53. [PubMed: 25043012]

95. Chamberlain PP, et al. Structure of the human cereblon-DDB1-lenalidomide complex reveals basis for responsiveness to thalidomide analogs. Nat Struct Mol Biol. 2014; 21:803-809. [PubMed: 25108355]

96. Winter GE, et al. Phthalimide conjugation as a strategy for in vivo target protein degradation. Science. 2015; 348:1376-1381. [PubMed: 25999370]

97. Koehler AN. A complex task? Direct modulation of transcription factors with small molecules. Curr Opin Chem Biol. 2010; 14:331-340. [PubMed: 20395165]

98. Wang T, et al. Genetic screens in human cells using the CRISPR-Cas9 system. Science. 2014; 343:80-84. [PubMed: 24336569]

99. Shalem O, et al. Genome-scale CRISPR-Cas9 knockout screening in human cells. Science. 2014; 343:84-87. [PubMed: 24336571]

100. Shi J, et al. Discovery of cancer drug targets by CRISPR-Cas9 screening of protein domains. Nature biotechnology. 2015; 33:661-667. 


\section{Trends}

TFs are commonly deregulated in the pathogenesis of human and are a major class of cancer cell dependencies. This makes TFs attractive targets for cancer therapy.

Drugs that target nuclear hormone receptors are among the most impactful targeted therapies in all of oncology. Insights into their mechanism of action and mechanisms of resistance have illuminated fundamental concepts of TF-targeting therapeutics.

Therapeutic targeting of general transcriptional cofactors can lead to remarkable efficacy in animal cancer models with surprisingly little toxicity to normal tissues.

Chemical targeting of TFs for proteolysis is among the few curative strategies in cancer therapeutics today and is an emerging approach to suppress intractable protein targets. 


\section{Outstanding Questions}

Can insights into the curative mechanisms of ATRA and $\mathrm{As}_{2} \mathrm{O}_{3}$ in APL inform how we might pursue disease-eradicating therapies in other types of cancer?

Will structural insight into TF-cofactor interaction surfaces inform the development of potent and selective small molecules that suppress oncogenic transcriptional circuits?

Why does chemical targeting of general components of the transcriptional apparatus lead to gene-specific transcriptional effects? Why are such agents selectively toxic to cancer cells relative to normal tissues?

Will oligonucleotide-based approaches (RNA interference, antisense oligonucleotides, DNA decoys) provide novel avenues to selectively target TFs in cancer?

Can cell-based screens be designed that reveal novel small molecules that convert oncogenic TFs into neosubstrates for ubiquitin conjugating enzymes?

What is the complete repertoire of essential TFs for the maintenance of each cancer type? How many TFs are required in a cancer-specific manner and would be suitable as therapeutic targets? How many TFs would lead to cancer-specific lethality if overexpressed or stabilized?

Can mapping of TF cistromes be implemented as a diagnostic test to guide the clinical management of cancer patients? Can mapping of TF dependencies in tumor specimens be performed to guide the use of TF-modulating therapeutics?

Can emerging insights into mechanisms of resistance to NHR-modulating therapies be utilized to develop combination drug regimens that improve overall survival? 


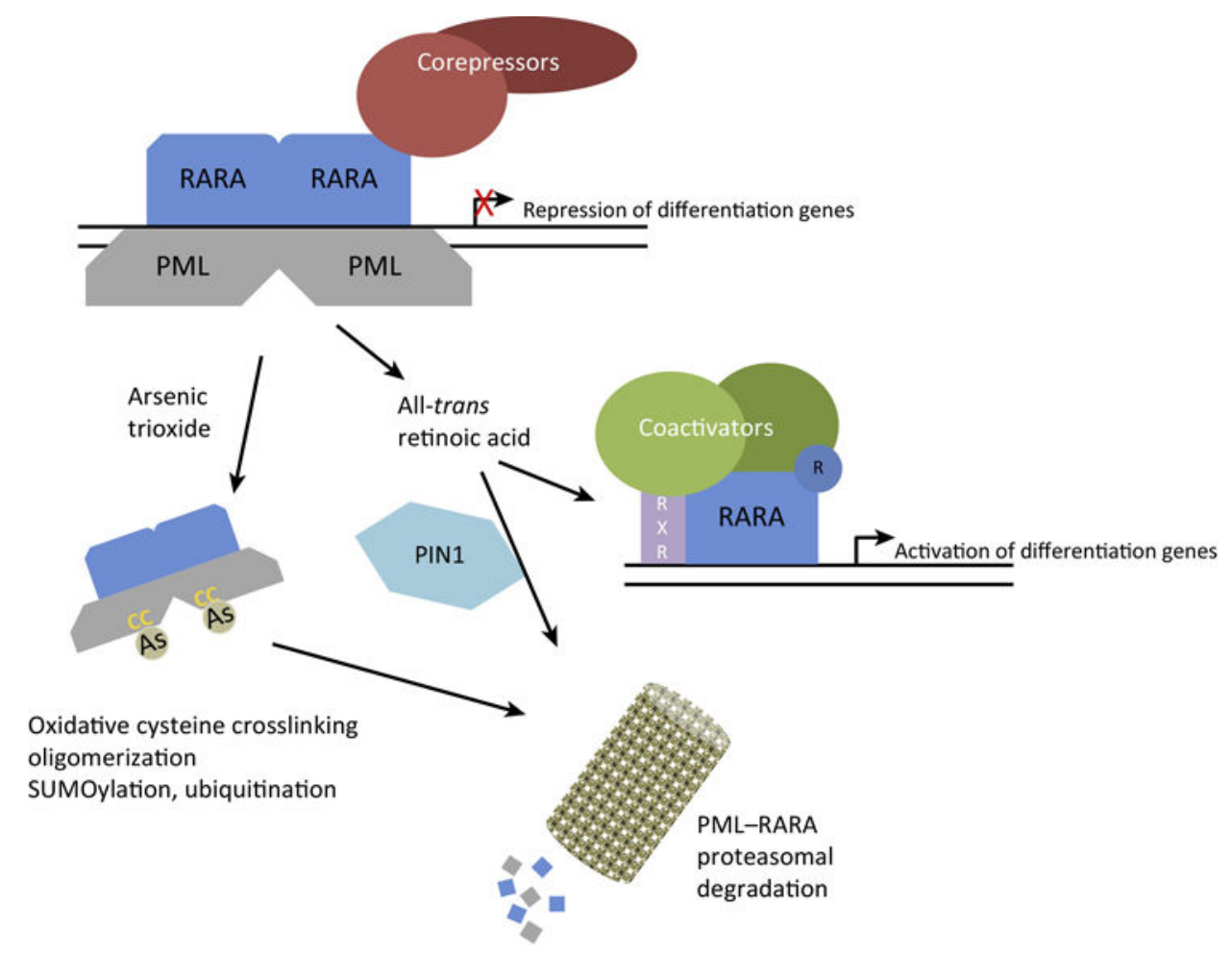

Figure 1.

Targeting of PML-RARA in Acute Promyelocytic Leukemia. The PML-RARA fusion protein binds to RARA sites in the genome, recruiting co-repressors and repressing RARtarget genes. All-trans retinoic acid (ATRA, labeled as R) binds to PML-RARA and switches it from a repressor of myeloid differentiation genes to an activator. ATRA also induces degradation of PML-RARA through a direct effect on the PIN1 prolyl isomerase. Arsenic trioxide also binds directly to the fusion protein and induces its degradation interacting with the zinc finger of the PML moiety. Arsenic leads to ROS production and subsequent disulfide crosslinking of the cysteines, resulting in oligomerization, SUMOylation, and subsequent ubiquitin-mediated proteolysis. Abbreviations: PIN1, peptidylprolyl cis/trans isomerase 1; PML, promyelocytic leukemia; RARA, retinoic acid receptor $a$; ROS, reactive oxygen species; SUMO, small ubiquitin-related modifier. 

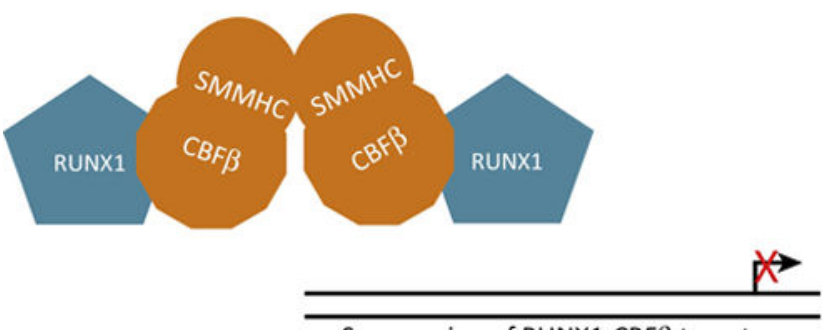

Suppression of RUNX1-CBF $\beta$ target genes (RUNX3, CEBPA, CSF1)

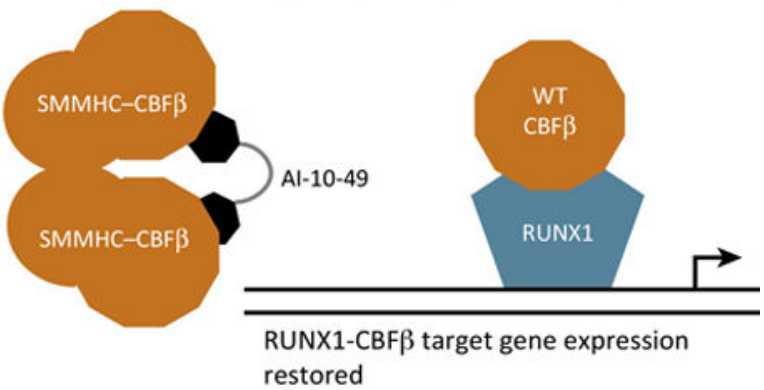

Figure 2.

Targeting of CBF $\beta-S M M H C$ in inv(16) AML. CBF $\beta$ is a cofactor for the TF RUNX1, which together regulate normal hematopoiesis. The inv(16) lesion that defines a subtype of AML results in the joining of smooth-muscle myosin heavy chain to CBF $\beta$. This fusion product is oligomeric and outcompetes wild-type CBF $\beta$ for RUNX1 binding. AI-10-49 is a bivalent inhibitor of CBF $\beta$-SMMHC that prevents its interaction with RUNX1, thus restoring the formation of RUNX1-CBF $\beta$ heterodimers. Abbreviations: AML, acute myeloid leukemia; CBF $\beta$, core-binding factor, $\beta$ subunit, RUNX, Runt-related transcription factor; SMMHC, myosin heavy chain 11 , smooth muscle. 


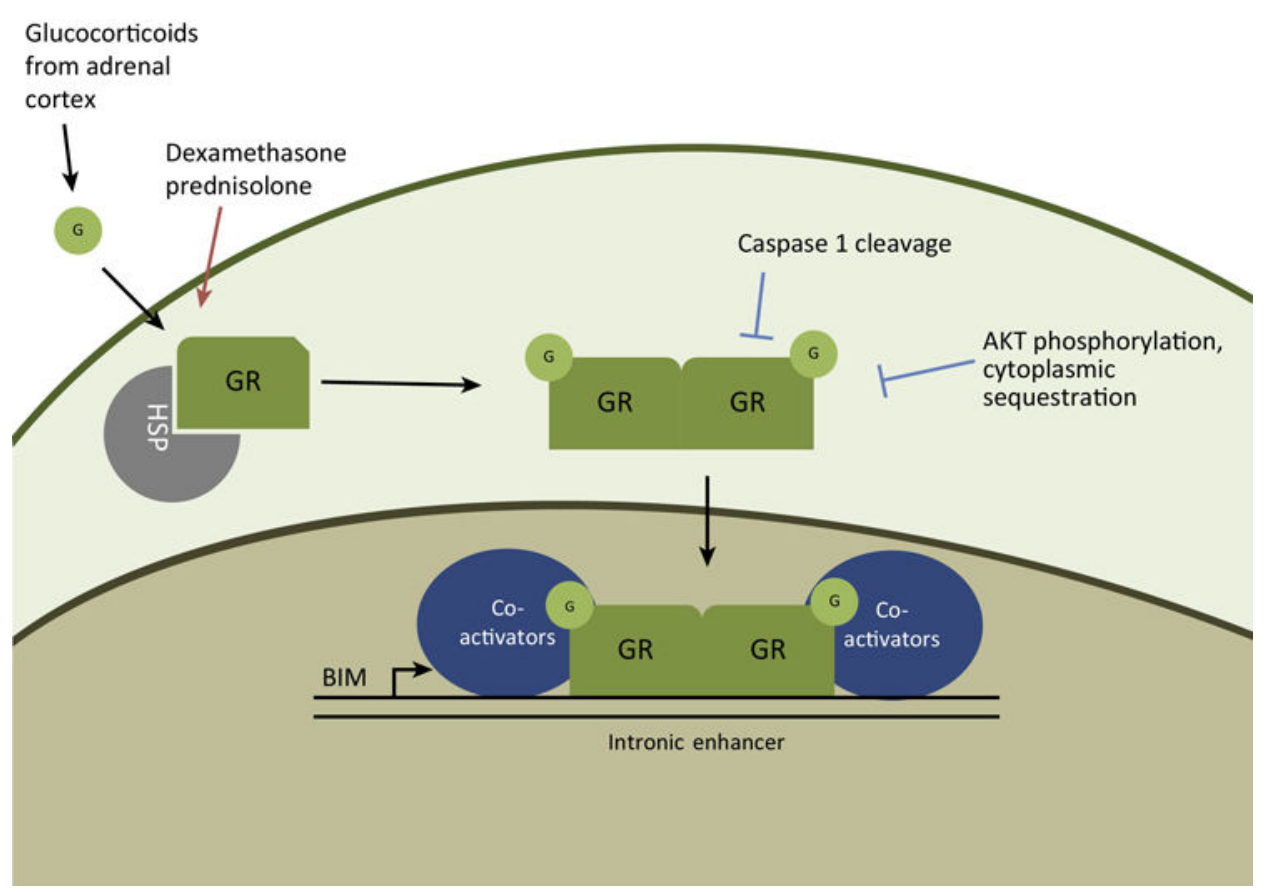

Figure 3.

Targeting the Glucocorticoid Receptor (GR) in Lymphoid Cancers. Endogenous glucocorticoids $(\mathrm{G})$ or synthetic glucocorticoids such as dexamethasone and prednisolone enter a leukemia cell and bind to the GR. Upon binding its ligand, GR is released from heat shock protein (HSP) chaperones, dimerizes, and enters the nucleus. Its key target genes for cancer therapy include the proapoptotic gene BIM (BCL2 interacting mediator of cell death). GR binds to a regulatory element in an intron of BIM, and recruits coactivators to drive expression of BIM to promote apoptosis of normal and neoplastic lymphoid cells. AKT, protein kinase $\mathrm{B}$. 
(A) Estrogens from

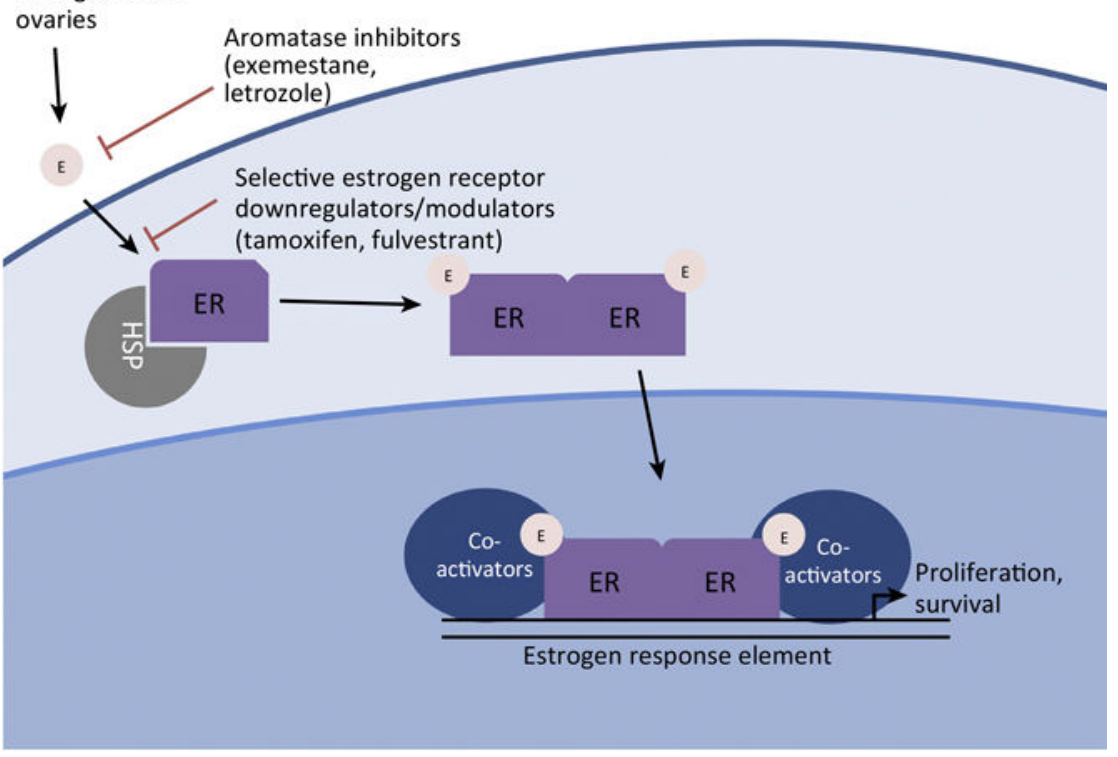

(B)

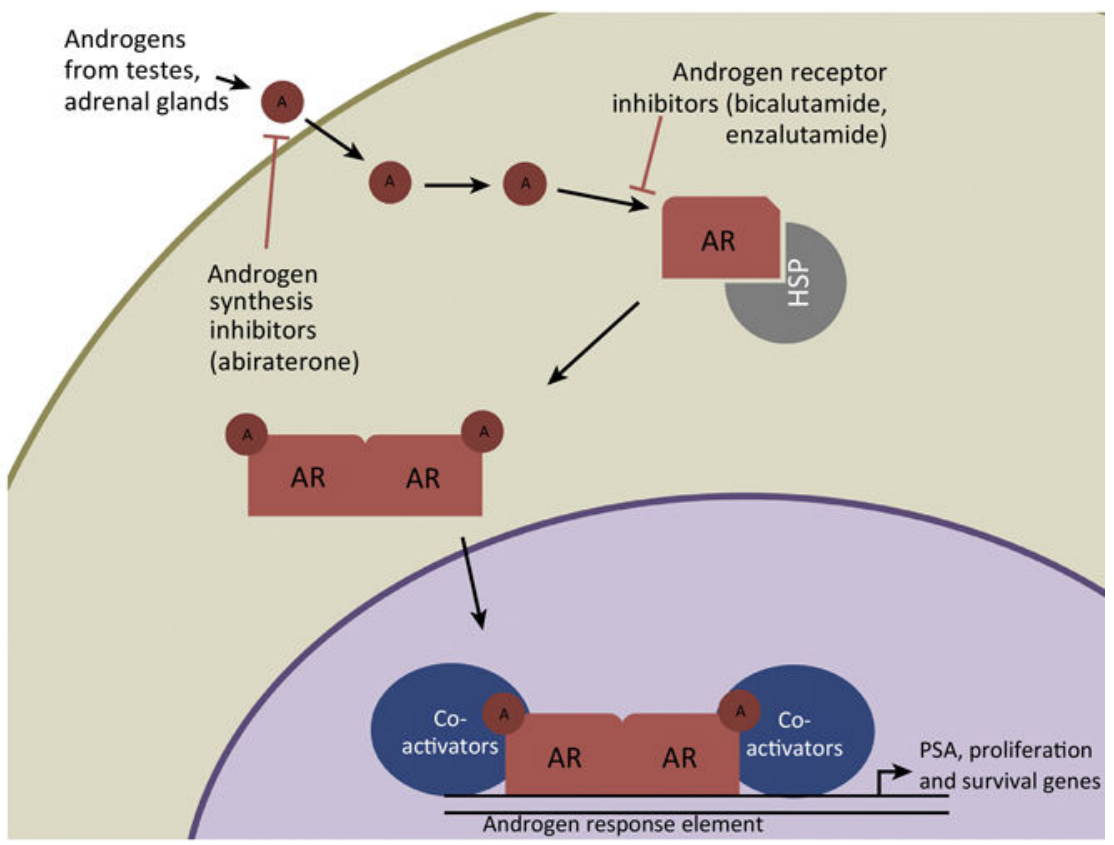

Figure 4.

Targeting the Estrogen and Androgen Receptors in Breast and Prostate Cancers,

Respectively. (A) Estrogens (E), primarily produced in the ovaries, enter a breast epithelial cell and bind to the estrogen receptor (ER). Upon binding its ligand, ER is released from heat shock protein (HSP) chaperones, dimerizes, and enters the nucleus. Here, it binds to estrogen response elements and recruits coactivators to regulate its target genes to promote the growth, proliferation, and survival of breast cells. Anti-estrogen therapy comes in two major modes: inhibitors of estrogen synthesis, such as the aromatase inhibitors exemestane and letrozole, and inhibitors of ER ligand binding, such as tamoxifen and fulvestrant. (B) Androgens (A) enter a prostate epithelial cell, are converted from testosterone to 
dihydrotestosterone, and bind to the androgen receptor (AR). Upon binding its ligand, AR is released from HSP chaperones, dimerizes, and enters the nucleus. It recruits coactivator proteins to androgen response elements in the genome, including those regulating the prostate cancer biomarker prostate-specific antigen (PSA), and promotes the growth, proliferation, and survival of prostate cells. Anti-androgen therapy falls principally into two categories: androgen synthesis inhibitors, such as the CYP17A1 inhibitor abiraterone, and inhibitors of ligand binding, such as bicalutamide and enzalutamide. 


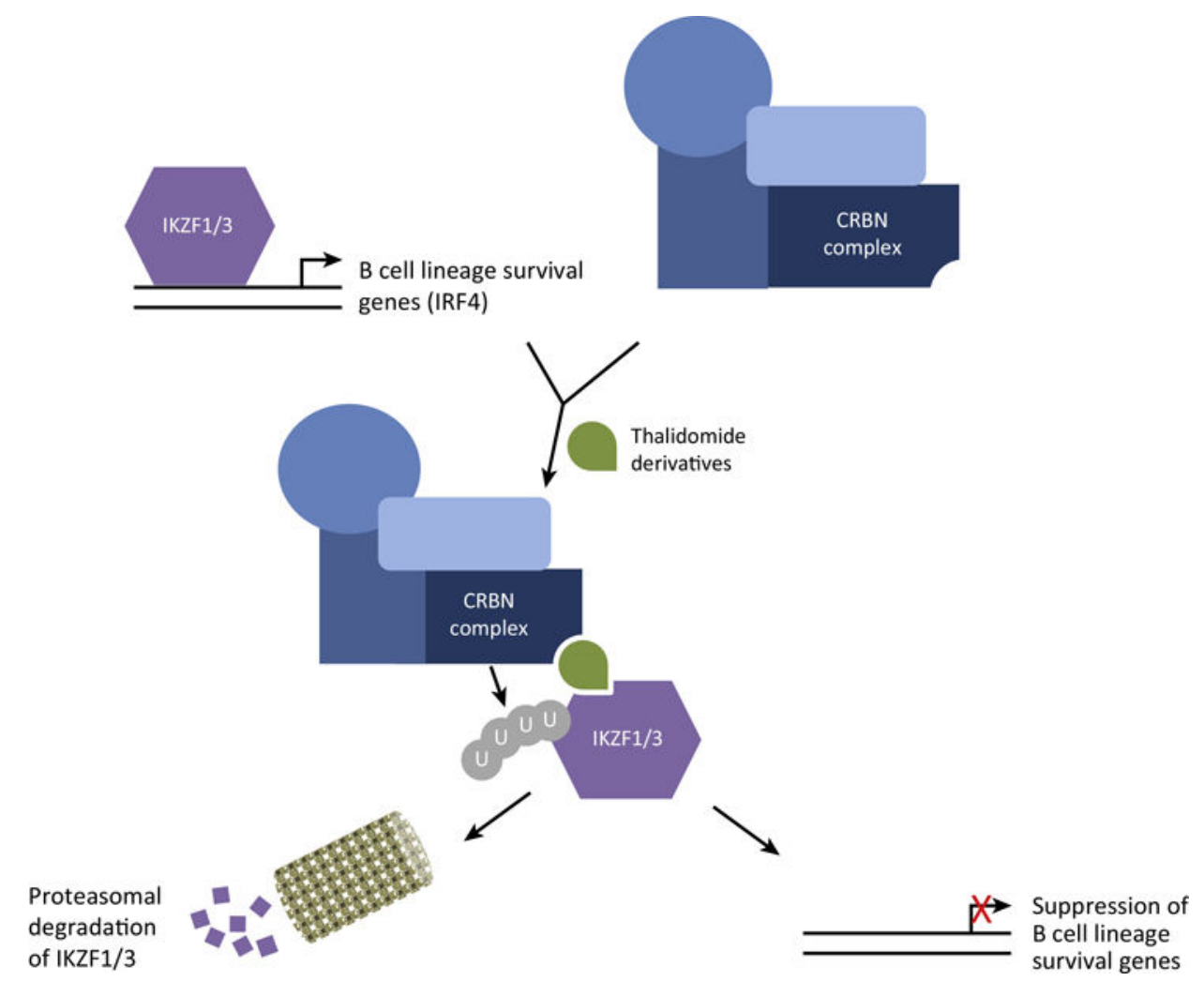

Figure 5.

Targeting Ikaros TFs with Phthalimide Induces Cereblon-Mediated Proteolysis. IKZF1 and IKZF3 are TFs that regulate B cell lineage transcriptional programs. Targets of IKZF1/3 in multiple myeloma include IRF4. Cereblon (CRBN) is an E3 ubiquitin ligase in a complex. Thalidomide and its derivatives pomalidomide and lenalidomide bind to cereblon, blocking its access to its normal ubiquitylation targets. These drugs additionally bind to IKZF1/3, thus recruiting them to the CRBN complex and resulting in their ubiquitylation (U) and proteasomal degradation as a neosubstrate. IKZF, Ikaros family zinc finger 1; IRF4 interferon regulatory factor 4 . 